\title{
Weber-like interactions and energy conservation
}

\author{
F. Bunchaft and S. Carneiro \\ Instituto de Física, Universidade Federal da Bahia \\ 40210-340, Salvador, BA, Brasil
}

\begin{abstract}
Velocity dependent forces varying as $k(\hat{\mathbf{r}} / r)\left(1-\mu \dot{r}^{2}+\gamma r \ddot{r}\right)$ (such as Weber force), here called Weber-like forces, are examined from the point of view of energy conservation and it is proved that they are conservative if and only if $\gamma=2 \mu$. As a consequence, it is shown that gravitational theories employing Weber-like forces cannot be conservative and also yield both the precession of the perihelion of Mercury as well as the gravitational deflection of light.
\end{abstract}

Key words: gravitational interaction, deflection of light, perihelion precession.

\section{Introduction}

One and a half century ago, when Weber [1] established the bases of his electrodynamics, the energy conservation arose as a central problem of the new theory since, for the first time, a velocity-dependent force law was stated for a basic interaction of nature:

$$
\mathbf{F}_{W}=\frac{q_{1} q_{2}}{4 \pi \epsilon_{0} r^{2}} \hat{\mathbf{r}}\left(1-\frac{1}{2 c^{2}} \dot{r}^{2}+\frac{1}{c^{2}} r \ddot{r}\right) .
$$

Here, $q_{1}, q_{2}$ are the electric charges, $\epsilon_{0}$ is the vacuum permittivity, $r=$ $|\mathbf{r}|=\left|\mathbf{r}_{1}-\mathbf{r}_{2}\right|$, the separation distance from $q_{2}$ to $q_{1}$, and $\hat{\mathbf{r}}=\mathbf{r} / r$; the dot signifies temporal derivation, and $c$ denotes simply the ratio between the electromagnetic and the electrostatics units of charge.

In order to face Helmholtz's criticism [2], Weber introduced for the first time a velocity-dependent potential energy 


$$
U_{W}=\frac{q_{1} q_{2}}{4 \pi \epsilon_{0} r}\left(1-\frac{1}{2 c^{2}} \dot{r}^{2}\right)
$$

and succeeded to prove that $\mathbf{F}_{W}$ is derivable from $U_{W}$.

Some years later Tisserand [3] proposed a Weber-like gravitational force law

$$
\mathbf{F}_{T}=-\frac{G m_{1} m_{2}}{r^{2}} \hat{\mathbf{r}}\left(1-\frac{1}{c^{2}} \dot{r}^{2}+\frac{2}{c^{2}} r \ddot{r}\right)
$$

derived from the Weber-like potential energy

$$
U_{T}=-\frac{G m_{1} m_{2}}{r}\left(1-\frac{1}{c^{2}} \dot{r}^{2}\right),
$$

where $m_{1}, m_{2}$ are the gravitational masses, $G$ is the gravitational constant, and $c$ stands also for the speed of light. With this force, Tisserand obtained $3 / 8$ of the then known value for the anomalous perihelion precession of Mercury and Levy [4], extending this potential energy, obtained the entire value for the precession.

In spite of its agreement with many theoretical and experimental results, Weber electrodynamics was replaced by the Maxwell-Lorentz field theory toward the end of the nineteenth century. And the interest in similar forces and potentials in gravitational theories also waned. Recently there has been a renewed interest in Weber electrodynamics in connection with important, but still controversial, experimental work $[5,6]$. And there has been renewed interest in Weber-like interactions in gravitational theories, such as Assis's Mach-like model [7]. With

$$
U_{A}=-\frac{G m_{1} m_{2}}{r}\left(1-\frac{3}{c^{2}} \dot{r}^{2}\right)
$$

and

$$
\mathbf{F}_{A}=-\frac{G m_{1} m_{2}}{r^{2}} \hat{\mathbf{r}}\left(1-\frac{3}{c^{2}} \dot{r}^{2}+\frac{6}{c^{2}} r \ddot{r}\right)
$$


Assis reobtained the correct expression for the perihelion precession.

More recently other theoretical Weber-like forces have been proposed to fit gravitational observations without, however, mentioning their conservative or nonconservative nature. Surprizingly enough, they are indeed generally nonconservative (as shown below). This means that the conservation of energy for Weber-like forces has not been adequately considered.

Raguza [8] extended Assis's theory by proposing the force

$$
\mathbf{F}_{R}=-\frac{G m_{1} m_{2}}{r^{2}} \hat{\mathbf{r}}\left(1-\frac{9}{c^{2}} \dot{r}^{2}+\frac{6}{c^{2}} r \ddot{r}\right)
$$

which not only yields the precession of the perihelion of Mercury but also accounts for the gravitational deflection of light grazing the sun.

Moreover Assis [9], trying to get a unification between gravitation and electromagnetism in a Weber-like framework, introduced a generalized electromagnetic potential energy from which he reobtained, to second order in $c^{-1}, \mathbf{F}_{W}$ (something already achieved by Phipps [10]); and, to fourth order in $c^{-1}$ (from the average electromagnetic interaction between neutral dipoles), the force

$$
\mathbf{F}_{A}^{*}=-\frac{G m_{1} m_{2}}{r^{2}} \hat{\mathbf{r}}\left(1-\frac{15}{c^{2}} \dot{r}^{2}+\frac{6}{c^{2}} r \ddot{r}\right)
$$

to be taken as the Weber-like gravitational force originating from electromagnetic interaction.

Nevertheless, the conservative, or not, nature of $\mathbf{F}_{R}$ and $\mathbf{F}_{A}^{*}$ has not been questioned at all. In doing so, we will not simply return to the old Helmholtz's requirement but, instead, propose the following inquiry: What is necessary and sufficient for a Weber-like force to be conservative? And, if so, what is the more general expression for the potential energy? This question, in all its generality, will be our main concern in this short article.

We will prove that any Weber-like force is conservative if and only if the coefficient $\gamma$ of the acceleration term is twice the coefficient $\mu$ of the velocity 
squared term (equation (9) below). The general form that any Weber-like potential must have is derived. It follows, in particular, that $\mathbf{F}_{R}$ and $\mathbf{F}_{A}^{*}$ are not conservative. A conservative Weber-like force can involve only one adjustable parameter; so a Weber-like force cannot simultaneously yield the gravitational deflection of light and also the precession of the perihelion of Mercury. This limitation does not end the matter: Generalized Weber-like forces, to be considered elsewhere, must also be examined.

\section{The form of conservative Weber-like forces}

Let us state the following definitions.

Def. 1. A force $\mathbf{F}$ between two particles will be said to be Weber-like when

$$
\mathbf{F}=\frac{k}{r^{2}} \hat{\mathbf{r}}\left(1-\mu \dot{r}^{2}+\gamma r \ddot{r}\right)
$$

where $k$ is a parameter that depends on the charges and characterises the nature of the interaction; $\mu \ll 1, \gamma \ll 1$ are positive constants referred to, respectivelly, the velocity and acceleration parameters.

Thus, a Weber-like force law has the following essential features: (i) It is relational, in the sense that it depends only on $r$ and its time derivatives. (ii) It is velocity and acceleration dependent, the velocity-dependent term being of opposite sign to the others. (iii) It tends to a Coulomb-Newton force law when $\dot{r} \rightarrow 0$ and $\ddot{r} \rightarrow 0$ (it reduces to such a force law in the static case). (iv) It obeys Newton's third law in strong form.

Remark 1. Consider a system of two bodies mutually interacting through forces $\mathbf{F}_{1}$ on body 1 and $\mathbf{F}_{2}$ on body 2 which obey Newton's third law in strong form. The work $\delta J$ done on the bodies during the time $d t$ by these 
forces and the kinetic energy $d T$ of the system are given by

$$
\delta J=\mathbf{F}_{1} \cdot d \mathbf{r}_{1}+\mathbf{F}_{2} \cdot d \mathbf{r}_{2}=d T=\mathbf{F}_{1} \cdot d\left(\mathbf{r}_{1}-\mathbf{r}_{2}\right)=\mathbf{F}_{1} \cdot d \mathbf{r}=F \hat{\mathbf{r}} \cdot d \mathbf{r}=F d r
$$

The forces are said conservative if and only if there exists some function $U$ of $r$ and its time derivatives, said the interaction potential energy, such that $d U=-\delta J$, that is, such that $d(T+U)=0$. Then

$$
F=-\frac{1}{\dot{r}} \frac{d U}{d t}
$$

This line of reasoning can be straightforwardly extended to many-body systems.

Def. 2. A function $U(r, \dot{r})$ will be said a Weber-like potential energy when the force derivable from $U$ is a (thus conservative) Weber-like force (9).

Remark 2. Let us observe that a Weber-like $U$ cannot be a function of higher order derivatives of $r$, since then extra terms, with derivatives of higher order then two, would appear in the derived force law, something which is excluded by Def. 1 .

The above definitions are sufficiently large to embrace all already known relational exact Weber-like models, as well as sufficiently appropriate to allow the unfolding of further investigations on generalized interactions which recover Weber-like forces in some $c^{-1}$ order of approximation. Here we will center our attention on Weber-like forces and Weber-like potentials".

The energy conservation criterion which we are searching for will be established by the following:

Theorem. Let $\mathbf{F}$ be a Weber-like force (9).

\footnotetext{
${ }^{1}$ In view of the Weber-Helmholtz controverse, may be it would be advisable to call weberian a conservative Weber-like force, and quasi-weberian a non-conservative one.
} 
(1) If $\mathbf{F}$ has $\gamma=2 \mu$, then it is conservative and its potential energy, apart an arbitrary additive constant, is given by

$$
U=\frac{k}{r}\left(1-\mu \dot{r}^{2}\right)
$$

(2) If $\mathbf{F}$ is conservative, then it must have $\gamma=2 \mu$ and its Weber-like potential, apart an arbitrary additive constant, must be given by (12).

Proof. The direct assertion is immediate since

$$
-\frac{1}{\dot{r}} \frac{d}{d t}\left[\frac{k}{r}\left(1-\mu \dot{r}^{2}\right)\right] \hat{\mathbf{r}}=\frac{k}{r^{2}} \hat{\mathbf{r}}\left(1-\mu \dot{r}^{2}+2 \mu r \ddot{r}\right) .
$$

Let us now demonstrate the inverse. In fact, if $\mathbf{F}$ is conservative and we take into account the previous Remarks, there must exist some function $U(r, \dot{r})$ such that

$$
\mathbf{F}=\frac{k}{r^{2}}\left(1+\gamma r \ddot{r}-\mu \dot{r}^{2}\right)=-\frac{1}{\dot{r}} \frac{d U}{d t}=-\frac{\partial U}{\partial r}-\frac{1}{\dot{r}} \frac{\partial U}{\partial \dot{r}} \ddot{r}
$$

That is,

$$
\ddot{r}\left(-\frac{1}{\dot{r}} \frac{\partial U}{\partial \dot{r}}-\frac{k}{r} \gamma\right)=\frac{\partial U}{\partial r}+\frac{k}{r^{2}}\left(1-\mu \dot{r}^{2}\right)
$$

This means that, once $U(r, \dot{r})$ is introduced in (15), both sides must be identical expressions in the variables $(r, \dot{r}, \ddot{r})$.

As the right-hand side of (15) does not contain $\ddot{r}$, it follows that both sides have to be identically constant. That is,

$$
\begin{aligned}
& \ddot{r}\left(-\frac{1}{\dot{r}} \frac{\partial U}{\partial \dot{r}}-\frac{k}{r} \gamma\right)=b, \\
& \frac{\partial U}{\partial r}+\frac{k}{r^{2}}\left(1-\mu \dot{r}^{2}\right)=b .
\end{aligned}
$$


Since the second factor of (16) does not contain $\ddot{r}$, the identity can be satisfied if and only if this factor is null, that is, if and only if $b=0$. In this case we have, by necessity, the following system of equations in $U$ :

$$
\begin{gathered}
-\frac{1}{\dot{r}} \frac{\partial U}{\partial \dot{r}}-\frac{k}{r} \gamma=0, \\
\frac{\partial U}{\partial r}+\frac{k}{r^{2}}\left(1-\mu \dot{r}^{2}\right)=0 .
\end{gathered}
$$

Equation (19) leads necessarily to

$$
U=\frac{k}{r}\left(1-\mu \dot{r}^{2}\right)+\phi(\dot{r}),
$$

where $\phi$ is a $C^{1}$-differentiable function of $\dot{r}$. Then, substituting (20) into (18), one gets

$$
\frac{1}{\dot{r}} \frac{d \phi}{d \dot{r}}=\frac{k}{r}(\gamma-2 \mu) .
$$

As in (21) the variables $(r, \dot{r})$ are separated, the two sides have to be identically constant, which leads to

$$
\begin{gathered}
\frac{k}{r}(\gamma-2 \mu)=d, \\
\frac{1}{\dot{r}} \frac{d \phi}{d \dot{r}}=d .
\end{gathered}
$$

Since $r$ is not a constant, (22) can be satisfied if and only if $\gamma=2 \mu$ (thus $d=0)$. This proves the first part of the reciprocal assertion. Besides, $d=0$ implies, through (23), that $\phi(\dot{r})$ must be an arbitrary constant and so (20) leads to the remaining part of the assertion.

Thus, the theorem means that any Weber-like interaction is conservative if and only if 


$$
\mathbf{F}=\frac{k}{r^{2}} \hat{\mathbf{r}}\left(1-\mu \dot{r}^{2}+2 \mu r \ddot{r}\right)
$$

and any potential energy $U$ is Weber-like if and only if it has the form (12).

Corollary 1. In any conservative Weber-like force law only one parameter can be independent.

Let us observe that, for the proof of the Theorem, it has never been necessary to restrict to $\mu \neq 0$ and/or $\gamma \neq 0$, so it is clear that this proof also implies the following

Corollary 2. A force

$$
\mathbf{F}=\frac{k}{r^{2}} \hat{\mathbf{r}}\left(1-\mu \dot{r}^{2}\right)
$$

is not conservative except for $\mu=0$.

This result is already contained in Helmholtz's mathematization of the principle of energy conservation and was the main basis for his first, erroneous, criticism of Weber's work [2]. Helmholtz was not aware that it is sufficient to add a suitable acceleration term to make the force conservative, something which Weber was the first to do, for his electromagnetic force.

\section{Weber-like forces and gravitational obser- vations}

We can now restate the results of Raguza [8], in the following manner:

(1) The observed precession of the perihelion of Mercury is obtained not only with Assis conservative force $\mathbf{F}_{A}$ (which leads to twice of the gravitational light deflection) but indeed with any Weber-like force with the 
coefficient of the acceleration term $\gamma_{A}=6 / c^{2}$, no matter the value of the coefficient $\mu$ of the velocity squared term.

(2) The gravitational deflection of light is given when $2 \gamma-\mu=3 / c^{2}$. Thus, to also yield the precession of the perihelion of Mercury, it must be $\mu=9 / c^{2}$, giving Raguza's force $\mathbf{F}_{R}$.

Now we can immediately add that $\mathbf{F}_{R}$ is non-conservative, since for this force $\gamma \neq 2 \mu$. In fact, as the conditions $\gamma=6 / c^{2}, 2 \gamma-\mu=3 / c^{2}$ and $\gamma=2 \mu$ are incompatible, we have shown the following:

Proposition. No conservative gravitational Weber-like force can yield simultaneously the precession of the perihelion of Mercury and the gravitational deflection of light.

By the way, let us observe that if we assume $\gamma=2 \mu$ and $2 \gamma-\mu=3 / c^{2}$, that is, energy conservation and light deflection, it results precisely Tisserand's parameters, which lead to $\gamma_{T} / \gamma_{A}=1 / 3$ of the anomalous perihelion precession.

\section{Conclusions and conjectures}

It has been shown here that any Weber-like conservative force must have a relationship between the two parameters $\mu$ and $\gamma$, namely $\mu=\gamma / 2$. It has been further shown that this requirement is not compatible with the inde-

pendent choices of $\mu$ and $\gamma$ necessary to yield the precession of the perihelion of Mercury and at the same time to yield the gravitational deflection of light.

On the other hand, if the Weber-like force were not to conserve energy, then the two body system could not be isolated; and an interaction with the external universe would have to be assumed of the order of $1 / c^{2}$. This nonconservative interaction could not be due to radiation: electromagnetic 
radiation reaction is only of the order of $1 / c^{3}$, and gravitational radiation reaction must also be presumed to be of order greater than the second.

Nevertheless, some ways out of this situation can be conjectured. One of them is to assume that Weber-like forces must be improved (in analogy to what Phipps has done, for other reasons, with Weber's electromagnetic force) by extension to conservative generalized forces reducible to Weber-like forces at order $c^{-2}$. Then, the Weber-like force would only be committed to fit low velocity tests, so that Raguzas's light deflection relation and force could be naturally dismissed and we simply return, for low velocities, to Assis conservative force.

With respect to Assis force $\mathbf{F}_{A}^{*}$, it arises as the part of order $c^{-4}$ of a generalized conservative electromagnetic force, through a theoretical model which contains many, independent, phenomenological assumptions which have assured perihelion precession $\left(\gamma_{A}^{*}=6 / c^{2}\right)$, but without energy conservation for $\mathbf{F}_{A}^{*}\left(\mu_{A}^{*}=15 / c^{2}\right)$. Nevertheless, there still exists the possibility that a critical revision could lead to changes on these assumptions suitable to obtain both results, that is, $\mathbf{F}_{A}^{*}=\mathbf{F}_{A}$.

\section{Acknowledgements}

We would like to thanks A.K.T. Assis for the reading of the first version of the manuscript, S. Raguza for an interesting discussion, and J.P. Wesley for useful suggestions.

\section{References}

[1] W. Weber, Abh. Leibniz. Ges. Leipzig (1846) 209; Werke, Vol. 3 (Springer, Berlin, 1893), p.25; Ann.Phys. 73 (1848) 193; Abh. Math. Phys. Kl. K. Sachs Gess. Wiss. 10 (1871). 
[2] H. Helmholtz, Über die Erhaltung der Kraft (Engelmann, Leipzig, 1847); Phil. Mag. 44 (1872) 530.

[3] M. Tisserand, C.R.Acad.Sc. (Paris) 75 (1872) 760; 111 (1890) 313.

[4] M. Levy, C.R.Acad.Sc. (Paris) 110 (1890) 72.

[5] P. Graneau, Ampere-Neumann Electrodynamics of Metals (Hadronic Press, Norramtum, Mass., 1985).

[6] J.P. Wesley, Found.Phys.Lett. 3 (1990) 443; 3 (1990) 471; 3 (1990) 586.

[7] A.K.T. Assis, Found.Phys.Lett. 2 (1989) 301.

[8] S. Raguza, Found.Phys.Lett. 5 (1992) 585.

[9] A.K.T. Assis, Can.J.Phys. 10 (1992) 330.

[10] T.E. Phipps Jr., Phys.Essays 3 (1990) 414. 